\title{
The Analytic Hierarchy Process without the Theory of Oskar Perron
}

\author{
Thomas L. Saaty \\ University of Pittsburgh \\ saaty@katz.pitt.edu
}

\section{Introduction}

We begin by honoring the name of Oskar Perron who proved a very powerful theorem in mathematics about real positive matrices. I regret not meeting Perron (1880-1975) before he died in Munich, Germany. My work on the Analytic Hierarchy Process with real positive reciprocal matrices $A=\left(a_{i j}\right), a_{i j}>0, a_{j i}=a_{i j}{ }^{-1}$ for all $i$ and $j$, leads to the principal eigenvalue and eigenvector without the need for invoking any of Perron's results. Note that the order of these reciprocal matrices is not much larger than 7x7. They involve entries whose values lie between 9 and 1/9. Perron proved that if $A=\left(a_{i j}\right)$ is an $n \times n$ real positive matrix then there is a positive real number $\lambda_{\max }$, called the Perron root or the Perron-Frobenius eigenvalue, such that $\lambda_{\max }$ is a simple eigenvalue of $A$ that is real and positive. All other eigenvalues $\lambda$ of $A$ (possibly complex) are strictly smaller than $\lambda_{\max }$ in absolute value, $|\lambda|<\lambda_{\max }$. There exists an eigenvector $w=\left(w_{1}, \ldots, w_{n}\right)$ of $A$ with eigenvalue $\lambda_{\text {max }}$ such that all components of $w$ are positive: $A w=\lambda_{\max } w, w_{i}>0$ for $1 \leq$ $i \leq n$. There also exists a positive left eigenvector $v: v^{T} A=\lambda_{\max } v^{T}, v_{i}>0$. Perron also proved that the principal eigenvector $w$ corresponding to $\lambda_{\max }$ can be obtained by raising the matrix $A$ to infinite powers.

Interestingly, the principal eigenvalue and its principal eigenvector can be found for a real reciprocal positive matrix of small order without Perron's theory. The principal eigenvalue and eigenvector can be obtained from the solution of a system of equations without using the powers of the matrix as does Perron. We observe that if we know either $\lambda_{\max }$ or $w$, we also know the other. If for example, we know $\lambda_{\max }$, we get $w$ by solving in the familiar way, the homogenous system of linear equations: $\sum_{j=1}^{n} a_{i j} w_{j}=\lambda_{\max } w_{i}, i=1, \ldots, n$. If we know $w$ then because of the normalization condition $\sum_{j=1}^{n} w_{i}=1$ in our case, we obtain after taking the sum on both sides of the equation with respect to $i$ and interchanging the sums on the left: $\sum_{i=1}^{n} w_{j} \sum_{j=1}^{n} a_{i j}=\lambda_{\max } \sum_{i=1}^{n} w_{i}=\lambda_{\max }$. In other words we obtain $\lambda_{\max }$ as the scalar product of the vector $w$ with the vector of column sums of the matrix $A$. If the matrix has real coefficient that are positive and if $w$ is real and positive then $\lambda_{\max }$ is real and positive. But we do not have the fact that it is a simple eigenvalue and that it dominates all other eigenvalues in modulus. 
We follow two routes to obtain the principal eigenvector $w$ : one by using the general idea of perturbation of the coefficients of a consistent matrix, which also involves reciprocal values in the transpose position, and the other by considering the graph theoretic concept of dominance along paths of different lengths leading to Cesaro summability. For us, applied to AHP reciprocal matrices, Cesaro summability says that the average of the normalized vector of the row sums of each power of a positive reciprocal matrix is equal to the normalized vector of the row sums of the limiting power of that matrix, which of course according to Perron is the principal eigenvector of that matrix. The latter again gives the same answer as the theory of Perron does without the need for Perron's logic whether that matrix is consistent or inconsistent by using perturbation arguments from the work of J. H. Wilkinson yields the principal eigenvalue and eigenvector in the limit.

\section{Consistent positive reciprocal matrices}

Assume that one is given $n$ stones, $A_{1}, \ldots, A_{n}$, with known weights $w_{1}, \ldots, w_{n}$, respectively, and suppose that a matrix of pairwise ratios is formed whose rows give the ratios of the weights of each stone with respect to all others. The paired comparisons process using actual measurements for the elements being compared takes the form:

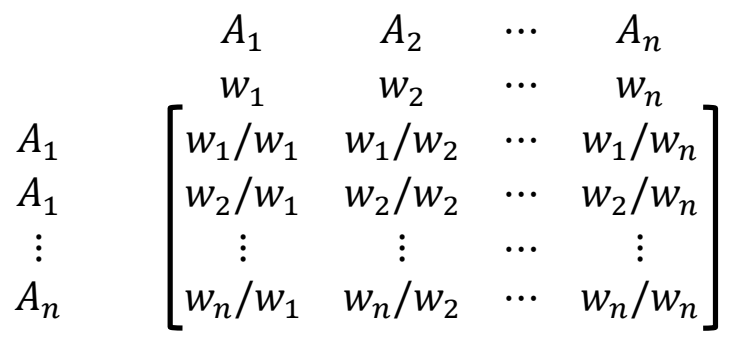

We note that we can recover the vector $w=\left(w_{1}, \ldots, w_{n}\right)$ by solving the system of equations defined by:

$$
A w=\left[\begin{array}{cccc}
w_{1} / w_{1} & w_{1} / w_{2} & \cdots & w_{1} / w_{n} \\
w_{2} / w_{1} & w_{2} / w_{2} & \cdots & w_{2} / w_{n} \\
\vdots & \vdots & \cdots & \vdots \\
w_{n} / w_{1} & w_{n} / w_{2} & \cdots & w_{n} / w_{n}
\end{array}\right]\left[\begin{array}{c}
w_{1} \\
w_{2} \\
\vdots \\
w_{n}
\end{array}\right]=n\left[\begin{array}{c}
w_{1} \\
w_{2} \\
\vdots \\
w_{n}
\end{array}\right]=n w
$$

Solving this homogeneous system of linear equations $A w=n w$ to find $w$ is a trivial eigenvalue problem, because the existence of a solution depends on whether or not $n$ is an eigenvalue of the characteristic equation of $A$. But $A$ has rank one and thus all its eigenvalues but one are equal to zero. The sum of the eigenvalues of a matrix is equal to its trace, the sum of its diagonal elements, which in this case is equal to $n$. Thus $n$ is the largest or the principal eigenvalue of $A$ and $w$ is its corresponding principal eigenvector that is positive and unique to within multiplication by a constant, and thus belongs to a ratio scale. We now know what must be done to recover the weights $w_{i}$, whether they are known in advance or not. 
Definition: An $n$ by $n$ matrix $A=\left(a_{i j}\right)$ is consistent if $a_{i j} a_{j k}=a_{i k}, i, j, k=1, \ldots, n$ holds among its entries.

We have for a consistent matrix $A^{k}=n^{k-1} A$. A consistent matrix always has the form $A=\left(\frac{w_{i}}{w_{j}}\right)$.

The consistent case has no need for the theorem of Perron to prove the existence of a largest real eigenvalue and a corresponding positive eigenvector nor that this vector is the limit to which powers of the matrix converge. Of course, real world reciprocal pairwise comparison matrices are very unlikely to be consistent unless they use actual measurement data.

Now we give a mathematical discussion to show why when a matrix is inconsistent, we still need the principal right eigenvector for our priority vector. It is clear that no matter what method we use to derive the weights $w_{i}$, we need to get them back as proportional to the expression $\sum_{j=1}^{n} a_{i j} w_{j} \quad i=1, \ldots, n$, that is, we must solve $\sum_{j=1}^{n} a_{i j} w_{j}=c w_{i} \quad i=1, \ldots, n$. Otherwise $\sum_{j=1}^{n} a_{i j} w_{j} \quad i=1, \ldots, n$ would yield another set of different weights and they in turn can be used to form new expressions $\sum_{j=1}^{n} a_{i j} w_{j} \quad i=1, \ldots, n$, and so on ad infinitum. Unless we solve the principal eigenvalue problem, our quest for priorities becomes meaningless.

We learn from the consistent case that what we get on the right is proportional to the sum on the left that involves the same scale used to weight the judgments that we are looking for. Thus we have the proportionality constant c. A better way to see this is to use the derived vector of priorities to weight each row of the matrix and take the sum. This yields a new vector of priorities (relative dominance of each element) represented in the comparisons. This vector can again be used to weight the rows and obtain still another vector of priorities. In the limit (if one exists), the limit vector itself can be used to weight the rows and get the limit vector back perhaps proportionately. Our general problem possibly with inconsistent judgments takes the form:

$$
A w=\left[\begin{array}{cccc}
1 & a_{12} & \cdots & a_{1 n} \\
1 / a_{12} & 1 & \cdots & a_{2 n} \\
\vdots & \vdots & \vdots & \vdots \\
1 / a_{12} & 1 / a_{12} & \cdots & 1
\end{array}\right]\left[\begin{array}{c}
w_{1} \\
w_{2} \\
\vdots \\
w_{n}
\end{array}\right]=c w
$$

This homogeneous system of linear equations $A w=c w$ has a solution $w$ if $\mathrm{c}$ is the principal eigenvalue of $A$. That this is the case can be shown using an argument that involves both left and right eigenvectors of $A$. Two vectors $x=\left(x_{1}, \ldots, x_{n}\right), y=\left(y_{1}, \ldots, y_{n}\right)$ are orthogonal if their scalar product $x_{1} y_{1}+\ldots+x_{n} y_{n}$ is equal to zero. It is known that any left eigenvector of a matrix 
corresponding to an eigenvalue is orthogonal to any right eigenvector corresponding to a different eigenvalue. This property is known as biorthogonality.

Theorem 1: For a given positive matrix $A$, the only positive vector $w$ and only positive constant $c$ that satisfy $A w=c w$, is a vector $w$ that is a positive multiple of the principal eigenvector of $A$, and the only such $c$ is the principal eigenvalue of $A$.

Proof: We know that the right principal eigenvector and the principal eigenvalue satisfy our requirements. We also know that the algebraic multiplicity of the principal eigenvalue is one, and that there is a positive left eigenvector of $A$ (call it $\mathrm{z}$ ) corresponding to the principal eigenvalue. Suppose there is a positive vector $y$ and a (necessarily positive) scalar $d$ such that $A y$ $=d y$. If $d$ and $c$ are not equal, then by biorthogonality $y$ is orthogonal to $z$, which is impossible since both vectors are positive. If $d$ and $c$ are equal, then $y$ and $w$ are dependent since $c$ has algebraic multiplicity one, and $y$ is a positive multiple of $w$. This completes the proof.

Next we show that order holds for a consistent matrix $A$. Element $A_{i}$ is said to dominate element $A_{j}$ in one step, if the sum of the entries in row $i$ of $A$ is greater than the sum of the entries in row $j$. It is convenient to use the vector $e=(1, \ldots, 1)^{\mathrm{T}}$ to express this dominance: Element $A_{i}$ dominates element $A_{j}$ in one step if $(A e)_{i}>(A e)_{j}$. An element can dominate another element in more than one step by dominating other criteria that in turn dominate the second criterion. Two-step dominance is identified by squaring the matrix and summing its rows, three-step dominance by cubing it, and so on. Thus, $A_{i}$ dominates $A_{j}$ in k steps if $\left(A^{k} e\right)_{i}>\left(A^{k} e\right)_{j}$. Element $A_{i}$ is said simply to dominate $A_{j}$ if entry $i$ of the vector obtained by averaging over the one step dominance vector, two step dominance vector, $\mathrm{k}$ step dominance vector and passing to the limit:

$$
\lim _{m \rightarrow \infty} \frac{1}{m} \sum_{i=1}^{m} A^{k} e / e^{T} A^{k} e
$$

is greater than its entry $j$. But this limit of weighted averages (the Cesaro sum) can be evaluated: We have for an $n$ by $n$ consistent matrix $A$ : $A^{k}=n^{k-1} A, A=\left(w_{i} / w_{j}\right)$ and the foregoing limit is simply the eigenvector $w$ normalized. In general, it is also true that the Cesaro sum converges to the same limit as does its kth term $A^{k} e / e^{T} A^{k} e$ that yields k step dominance.

Here we see that the requirement for rank takes on the particular form of the principal eigenvector. We will not assume it for the inconsistent case but prove its necessity again for that more general case.

We now develop a necessary and sufficient condition for rank preservation. For emphasis, recall from graph theory that an element $a_{i j}^{(m)}$ of $A^{m}$ gives cumulative dominance of the $i$ th element over the $j$ th element along all chains of length $m$. That is precisely how one measures the consistency relation between that row and each column. In fact when $A$ is consistent we have from $A^{m}=n^{m-1} A$ that the entries of $A^{m}$ and those of $A$ differ by a constant thus maintaining consistency. 
In general, consider $A^{m}=\left(a_{i j}^{(m)}\right)$.

Theorem 1. For a positive reciprocal matrix $A$

$$
\lim _{m \rightarrow \infty} \frac{a_{i k}^{(m)}}{\sum_{i-1}^{n} a_{i k}^{(m)}}=\lim _{m \rightarrow \infty} \frac{a_{i s}^{(m)}}{\sum_{i-1}^{n} a_{i s}^{(m)}}, \quad k, s=1,2, \ldots, n .
$$

Proof. Let $B=N A N^{-1}$ be the Jordan canonical form of $A$ given by

$$
B=\left[\begin{array}{llll}
\lambda_{1} & & & \\
& B_{2} & & \\
& & \ddots & \\
& & & B_{r}
\end{array}\right]
$$

where $\lambda_{1} \equiv \lambda_{\max }$, and $B_{p}, p=2,3, \ldots, r$ is the $n_{p} \times n_{p}$ Jordan block defined by

$$
B=\left[\begin{array}{cccccc}
\lambda_{p} & 0 & 0 & \cdots & 0 & 0 \\
1 & \lambda_{p} & 0 & & 0 & 0 \\
0 & 1 & \lambda_{p} & & 0 & 0 \\
\vdots & & & \ddots & & \vdots \\
0 & 0 & 0 & \cdots & 1 & \lambda_{p}
\end{array}\right],
$$

where $\lambda_{p}, p=2, \ldots, r$ are distinct eigenvalues with multiplicities $n_{2}, \ldots, n_{r}$ respectively, and

$\sum_{p=2}^{r} n_{p}=n-1$. We have $A=N^{-1} B N$ and $A^{m}=N^{-1} B^{m} B$, where $B^{m}$ is given by

$$
B_{p}=\left[\begin{array}{cccc}
\lambda_{1}^{m} & 0 & \cdots & 0 \\
0 & B_{2}^{m} & & 0 \\
\vdots & & \ddots & \vdots \\
0 & 0 & \cdots & B_{r}^{m}
\end{array}\right]
$$

Let us denote $N^{-1} \equiv D=\left(d_{i j}\right)$ and $N=\left(n_{i j}\right)$. We have

$$
A^{m} D B^{m} N=\left[\begin{array}{cccccccc}
n_{11} d_{11} \lambda_{1}^{m}+ & \cdots & , & n_{12} d_{11} \lambda_{1}^{m}+ & \cdots & , \ldots, & n_{1 n} d_{11} \lambda_{1}^{m}+ & \ldots \\
n_{11} d_{21} \lambda_{1}^{m}+ & \cdots & , & n_{12} d_{21} \lambda_{1}^{m}+ & \cdots & , \ldots, & n_{1 n} d_{21} \lambda_{1}^{m}+ & \ldots \\
& & \vdots & & \ddots & & \vdots \\
n_{11} d_{n 1} \lambda_{1}^{m}+ & \cdots & , & n_{12} d_{n 1} \lambda_{1}^{m}+ & \cdots & , \ldots, & n_{1 n} d_{n 1} \lambda_{1}^{m}+ & \ldots
\end{array}\right]
$$

Let $e=(1,1, \ldots, 1)^{T}=a_{1} w_{1}+\cdots+a_{r} w_{r}$, where $w_{p}$ is the principal right eigenvector corresponding to $\lambda_{p}$. We have

$$
\begin{gathered}
e^{T} A^{m}=a_{1} \lambda_{1}^{m} w_{1}^{T}+\cdots+a_{r} \lambda_{1}^{m} w_{1}^{T} \\
=\left(n_{11} \sum_{i=1}^{n} d_{11} \lambda_{1}^{m}+\cdots, \ldots, n_{1 n} \sum_{i=1}^{n} d_{11} \lambda_{1}^{m}+\cdots\right) .
\end{gathered}
$$


Given two columns of $A, k$ and $s$ we have

$$
\frac{a_{i k}^{(m)}}{\sum_{i=1}^{n} a_{i k}^{(m)}}=\frac{n_{1 k} d_{i 1} \lambda_{1}^{m}+\cdots}{n_{1 k} \sum_{i=1}^{n} d_{i 1} \lambda_{1}^{m}+\cdots} \quad \text { and } \quad \frac{a_{i s}^{(m)}}{\sum_{i=1}^{n} a_{i s}^{(m)}}=\frac{n_{1 s} d_{i 1} \lambda_{1}^{m}+\cdots}{n_{1 s} \sum_{i=1}^{n} d_{i 1} \lambda_{1}^{m}+\cdots}
$$

Since both numerators and denominators are polynomials in $\lambda_{p}^{m}, p=1,2, \ldots, r$, and $\lambda_{1}=\lambda_{\text {max }}>\left|\lambda_{p}\right|, p \neq 1$, we have for the ith entries of two arbitrary columns $k$ and $s$

$$
\lim _{m \rightarrow \infty} \frac{a_{i k}^{(m)}}{\sum_{i=1}^{n} a_{i k}^{(m)}}=\lim _{m \rightarrow \infty} \frac{a_{i s}^{(m)}}{\sum_{i-1}^{n} a_{i s}^{(m)}}=\frac{d_{i 1}}{\sum_{i-1}^{n} d_{i 1}} .
$$

Definition. A positive matrix $A$ is said to be $m$-dominant if there is an $m_{0}$ such that for $m \geqslant m_{n}$ either $a_{i k}^{(m)} \geqslant a_{i \prime k}^{(m)}$ or $a_{i k}^{(m)} \leqslant a_{i \prime k}^{(m)}$ for all $k$ and for any pair $i$ and $i^{\prime}$.

COROLlARY. A positive reciprocal matrix is asymptotically m-dominant. ${ }^{2}$

Proof. We have from Theorem 1 that the normalized columns of $A^{m}$ - are the same in the limit. Since the elements in each row are identical, the result follows by choosing $m_{0}$ to be the maximum of its values for each pair of rows.

We now show that for an inconsistent matrix $A$, rank is determined in terms of the powers of $A$. To do this we demonstrate that there is a method of estimating $x$ which coincides with the normalized limiting columns of $A$. This method is precisely the eigenvalue method.

Theorem 2. $\quad \lim _{m \rightarrow \infty}\left(\frac{a_{i k}^{(m)}}{\sum_{i=1}^{n} a_{i k}^{(m)}}\right)=w_{i}, i=1,2, \ldots, n$.

Proof. From $\lim _{m \rightarrow \infty}\left(\frac{A^{m} e}{|| A^{m} \|}\right)=w$, we have $w_{i}=\lim _{m \rightarrow \infty}\left(\frac{1}{\left\|A^{m}\right\|}\right) \sum_{k=1}^{n} a_{i k}^{(m)}$.

Multiplying and dividing $a_{i k}^{(m)}$ by $\sum_{k=1}^{n} a_{i k}^{(m)}$ we have on distributing the limit with respect to the finite sum

$$
\begin{gathered}
w_{i}=\sum_{k=1}^{n} \lim _{m \rightarrow \infty}\left[\frac{a_{i k}^{(m)}}{|| A^{m} \|}, \frac{\sum_{i=1}^{n} a_{i k}^{(m)}}{\sum_{i=1}^{n} a_{i k}^{(m)}}\right] \\
=\sum_{k=1}^{n}\left[\lim _{m \rightarrow \infty} \frac{a_{i k}^{(m)}}{\sum_{i=1}^{n} a_{i k}^{(m)}}\right]\left[\lim _{m \rightarrow \infty} \frac{\sum_{i=1}^{n} a_{i k}^{(m)}}{\left\|A^{m}\right\|}\right] .
\end{gathered}
$$

By Theorem $1 \lim _{m \rightarrow \infty} \frac{a_{i k}^{(m)}}{\sum_{i=1}^{n} a_{i k}^{(m)}}$ is the same constant for all $k$ hence we have

$$
w_{i}=\lim _{m \rightarrow \infty} \frac{a_{i k}^{(m)}}{\sum_{i=1}^{n} a_{i k}^{(m)}} \sum_{k=1}^{n}\left[\lim _{m \rightarrow \infty} \frac{\sum_{i=1}^{n} a_{i k}^{(m)}}{\left\|A^{m}\right\|}\right] .
$$

Since ||$A^{m} \|=\sum_{i=1}^{n} \sum_{k=1}^{n} a_{i k}^{(m)}$, the proof is complete.

There is a natural way to derive the rank order of a set of alternatives from a pairwise comparison matrix $A$. The rank order of each alternative is the relative proportion of its 
dominance over the other alternatives. This is obtained by adding the elements in each row in $A$ and dividing by the total. However, $A$ only captures the dominance of one alternative over each other in one step. But an alternative can dominate a second by first dominating a third alternative and then the third dominates the second. Thus, the first alternative dominates the second in two steps (along a path of length two). It is known that the result for dominance in two steps is obtained by squaring the pairwise comparison matrix. Similarly dominance can occur in three steps, four steps and so on, the value of each obtained by raising the matrix to the corresponding power. The rank order of an alternative is the average of the relative values for dominance in one step, two steps, and so on. We show below that when we take this infinite series of dominance along paths of length one, two, three, and so on and calculate its limiting value we obtain precisely the principal right eigenvector of the matrix $A$. This demonstrates that the eigenvector is derived deductively to obtain a relative scale among $n$ alternatives from their matrix of pairwise comparisons. It is the desired solution because it preserves rank order rather than a convenient criterion introduced for minimization purposes.

Theorem 3. The relative dominance of an alternative is given by the solution of the eigenvalue problem $A w=\lambda_{\max } w$.

by

Proof. The relative dominance of an alternative along all paths of length $k \leqslant m$ is given

Let

$$
\frac{1}{m} \sum_{k=1}^{m} \frac{A^{k} e}{e^{T} A^{k} e} .
$$

and

$$
s_{k}=\frac{A^{k} e}{e^{T} A^{k} e}
$$

$$
t_{m}=\frac{1}{m} \sum_{k=1}^{m} s_{k} .
$$

Note that $\lim _{m \rightarrow \infty} t_{m}<\infty$. This is a consequence of a theorem due to G. H. Hardy [1949] which gives necessary and sufficient conditions for a transformation of a convergent sequence to also be convergent. Let $T$ be such a transformation mapping

$$
\left(s_{1}, \ldots, s_{m}\right) \rightarrow t_{m}=\sum_{k=1}^{\infty} c_{m, k} s_{k} .
$$

$T$ is regular if $t_{m} \rightarrow s$ as $m \rightarrow \infty$ whenever $s_{k} \rightarrow s$ as $\mathrm{k} \rightarrow \infty$. It is known (Hardy, 1949) that $T$ is regular if and only if the following conditions hold:

(1) $\sum_{k=1}^{\infty}\left|c_{m, k}\right|<H$ (independent of $m$ ),

(2) $c_{m, k} \rightarrow \delta_{k}$ for each $k$, when $m \rightarrow \infty$,

(3) $\sum_{k=1}^{\infty} c_{m, k} \rightarrow \delta$ when $m \rightarrow \infty$,

(4) $\delta_{k}=0$ for each $k$,

(5) $\delta=1$.

Here, 
Thus, we have

$$
c_{m, k}= \begin{cases}\frac{1}{m} & \text { for } 1 \leqslant k \leqslant m \\ 0 & \text { for } k>m .\end{cases}
$$

(1) $\sum_{k=1}^{\infty}\left|c_{m, k}\right|=\sum_{k=1}^{m}\left|\frac{1}{m}\right|=1$,

(2) $c_{m, k}=\frac{1}{m} \rightarrow 0$ as $m \rightarrow \infty$. Hence (4) $\delta_{k}=0$ for each $k$,

(3) $\sum_{k=1}^{\infty} c_{m, k}=\sum_{k=1}^{\infty}\left(\frac{1}{m}\right)=1$ and hence (5) $\delta=1$.

It follows that $T$ is regular. Since $s_{k}=\frac{A^{k} e}{e^{T} A^{k} e} \rightarrow w$ as $k \rightarrow \infty$ (Saaty, 1980), where $w$ is the principal right eigenvector of $A$, we have

$$
t_{m}=\frac{1}{m} \sum_{k=1}^{m} \frac{A^{k} e}{e^{T} A^{k} e} \rightarrow w \quad \text { as } \quad m \rightarrow \infty
$$

In input/output analysis in economics multipliers are traced by raising the input/output matrix to higher and higher powers and taking their sums to obtain the overall impact of each sector of the economy on every other sector.

Still another argument can be constructed from Theorem 1 because for large $m$ the normalized columns of $A^{m}$ are the same and converge to the principal eigenvector.

Theorem 4: The Cesaro sum $\lim _{m \rightarrow \infty} \frac{1}{m} \sum_{k=1}^{m} \frac{A^{k} e}{e^{T} A^{k} e}$ is the principal right eigenvector of $A$. Proof:

By Theorem 3 of Saaty and Vargas (1984) we know that $\lim _{m \rightarrow \infty} \frac{1}{m} \sum_{k=1}^{m} \frac{A^{k} e}{e^{T} A^{k} e}=\lim _{k \rightarrow \infty} \frac{A^{k} e}{e^{T} A^{k} e}$.

Multiplying $\lim _{m \rightarrow \infty} \frac{1}{m} \sum_{k=1}^{m} \frac{A^{k} e}{e^{T} A^{k} e}$ by $A$ on the left we have

$$
\begin{gathered}
A\left(\lim _{m \rightarrow \infty} \frac{1}{m} \sum_{k=1}^{m} \frac{A^{k} e}{e^{T} A^{k} e}\right)=A\left(\lim _{k \rightarrow \infty} \frac{A^{k} e}{e^{T} A^{k} e}\right) \\
=\left(\lim _{k \rightarrow \infty} \frac{e^{T} A^{k+1} e}{e^{T} A^{k} e}\right)\left(\lim _{k \rightarrow \infty} \frac{A^{k+1} e}{e^{T} A^{k+1} e}\right)=\left(\lim _{k \rightarrow \infty} \frac{e^{T} A^{k+1} e}{e^{T} A^{k} e}\right)\left(\lim _{m \rightarrow \infty} \frac{1}{m} \sum_{k=1}^{m} \frac{A^{k} e}{e^{T} A^{k} e}\right)
\end{gathered}
$$

There is a vector $y=\lim _{m \rightarrow \infty} \frac{1}{m} \sum_{k=1}^{m} \frac{A^{k} e}{e^{T} A^{k} e}$ and a constant $d=\lim _{k \rightarrow \infty} \frac{e^{T} A^{k+1} e}{e^{T} A^{k} e}$ such that $A y=d y$. Under the assumption that $A$ has $r$ distinct eigenvalues $\lambda_{1}, \ldots, \lambda_{r}$ with multiplicities $n_{1}, \ldots, n_{r}$, respectively, by using the Jordan canonical form of A we can write $A=N^{-1} B N$ where $\mathrm{N}$ is an invertible matrix and

$$
B=\left[\begin{array}{cccc}
B_{1} & 0 & \cdots & 0 \\
0 & B_{2} & \cdots & 0 \\
\vdots & \vdots & \ddots & \vdots \\
0 & 0 & \cdots & B_{r}
\end{array}\right] \text { and } B_{p}=\left[\begin{array}{cccccc}
\lambda_{p} & 0 & 0 & 0 & \cdots & 0 \\
1 & \lambda_{p} & 0 & 0 & \cdots & 0 \\
0 & 1 & \lambda_{p} & 0 & \cdots & 0 \\
0 & 0 & 1 & \lambda_{p} & \ddots & 0 \\
\vdots & \vdots & \vdots & \ddots & \ddots & \vdots \\
0 & 0 & 0 & \cdots & 1 & \lambda_{p}
\end{array}\right], p=1, \ldots, r
$$


We have $A^{k}=N^{-1} B^{k} N, B^{k}=\left[\begin{array}{cccc}B_{1}^{k} & 0 & \cdots & 0 \\ 0 & B_{2}^{k} & \cdots & 0 \\ \vdots & \vdots & \ddots & \vdots \\ 0 & 0 & \cdots & B_{r}^{k}\end{array}\right]$ and

$B_{p}^{k}=\left[\begin{array}{cccccc}\lambda_{p}^{k} & 0 & 0 & 0 & \cdots & 0 \\ k \lambda_{p}^{k-1} & \lambda_{p}^{k} & 0 & 0 & \cdots & 0 \\ \frac{k(k-1)}{2 !} \lambda_{p}^{k-2} & k \lambda_{p}^{k-1} & \lambda_{p}^{k} & 0 & \cdots & 0 \\ \frac{k(k-1)(k-2)}{3 !} \lambda_{p}^{k-3} & \frac{k(k-1)}{2 !} \lambda_{p}^{k-2} & k \lambda_{p}^{k-1} & \lambda_{p}^{k} & \ddots & 0 \\ \vdots & \vdots & \vdots & \ddots & \ddots & \vdots \\ \frac{k(k-1) \cdots\left(k-n_{p}+1\right)}{\left(n_{p}-1\right) !} \lambda_{p}^{k-n_{p}+1} & \frac{k(k-1) \cdots\left(k-n_{p}+2\right)}{\left(n_{p}-2\right) !} \lambda_{p}^{k-n_{p}+2} & \frac{k(k-1) \cdots\left(k-n_{p}+3\right)}{\left(n_{p}-3\right) !} \lambda_{p}^{k-n_{p}+3} & \cdots & k \lambda_{p}^{k-1} & \lambda_{p}^{k}\end{array}\right]$

Denoting $N^{-1}=D=\left(d_{i j}\right)$ and $N=\left(n_{i j}\right)$ we can write $A^{k}=N^{-1} B^{k} N=D B^{k} N$. The first $n_{1}$ columns of $A^{k}$ are given by

$$
D_{n_{1}} B_{1}^{k}=\left(\left[\begin{array}{c}
d_{11} \lambda_{1}^{k}+d_{12}\left(\begin{array}{l}
k \\
1
\end{array}\right) \lambda_{1}^{k-1}+\cdots+d_{1 n_{1}}\left(\begin{array}{c}
k \\
n_{1}-1
\end{array}\right) \lambda_{1}^{k-n_{1}+1} \\
d_{21} \lambda_{1}^{k}+d_{22}\left(\begin{array}{c}
k \\
1
\end{array}\right) \lambda_{1}^{k-1}+\cdots+d_{2 n_{1}}\left(\begin{array}{c}
k \\
n_{1}-1
\end{array}\right) \lambda_{1}^{k-n_{1}+1} \\
\vdots \\
d_{n 1} \lambda_{1}^{k}+d_{n 2}\left(\begin{array}{c}
k \\
1
\end{array}\right) \lambda_{1}^{k-1}+\cdots+d_{n n_{1}}\left(\begin{array}{c}
k \\
n_{1}-1
\end{array}\right) \lambda_{1}^{k-n_{1}+1}
\end{array}\right]\left[\begin{array}{c}
d_{12} \lambda_{1}^{k}+d_{13}\left(\begin{array}{l}
k \\
1
\end{array}\right) \lambda_{1}^{k-1}+\cdots+d_{1 n_{1}}\left(\begin{array}{c}
k \\
n_{1}-2
\end{array}\right) \lambda_{1}^{k-n_{1}+2} \\
d_{22} \lambda_{1}^{k}+d_{23}\left(\begin{array}{c}
k \\
1
\end{array}\right) \lambda_{1}^{k-1}+\cdots+d_{2 n_{1}}\left(\begin{array}{c}
k \\
n_{1}-2
\end{array}\right) \lambda_{1}^{k-n_{1}+2} \\
\vdots \\
d_{n 2} \lambda_{1}^{k}+d_{n 3}\left(\begin{array}{c}
k \\
1
\end{array}\right) \lambda_{1}^{k-1}+\cdots+d_{n n_{1}}\left(\begin{array}{c}
k \\
n_{1}-2
\end{array}\right) \lambda_{1}^{k-n_{1}+2}
\end{array}\right], \ldots,\left(\begin{array}{c}
d_{1 n_{1}} \lambda_{1}^{k} \\
d_{2 n_{1}} \lambda_{1}^{k} \\
\vdots \\
d_{n n_{1}} \lambda_{1}^{k}
\end{array}\right]\right)
$$

The next $n_{2}$ columns are given by

$$
D_{n_{1}+1, n_{2}} B_{1}^{k}=\left(\left[\begin{array}{c}
d_{11} \lambda_{1}^{k}+d_{12}\left(\begin{array}{l}
k \\
1
\end{array}\right) \lambda_{1}^{k-1}+\cdots+d_{1 n_{1}}\left(\begin{array}{c}
k \\
n_{1}-1
\end{array}\right) \lambda_{1}^{k-n_{1}+1} \\
d_{21} \lambda_{1}^{k}+d_{22}\left(\begin{array}{l}
k \\
1
\end{array}\right) \lambda_{1}^{k-1}+\cdots+d_{2 n_{1}}\left(\begin{array}{c}
k \\
n_{1}-1
\end{array}\right) \lambda_{1}^{k-n_{1}+1} \\
\vdots \\
d_{n 1} \lambda_{1}^{k}+d_{n 2}\left(\begin{array}{c}
k \\
1
\end{array}\right) \lambda_{1}^{k-1}+\cdots+d_{n n_{1}}\left(\begin{array}{c}
k \\
n_{1}-1
\end{array}\right) \lambda_{1}^{k-n_{1}+1}
\end{array}\right]\left[\begin{array}{c}
d_{12} \lambda_{1}^{k}+d_{13}\left(\begin{array}{l}
k \\
1
\end{array}\right) \lambda_{1}^{k-1}+\cdots+d_{1 n_{1}}\left(\begin{array}{c}
k \\
n_{1}-2
\end{array}\right) \lambda_{1}^{k-n_{1}+2} \\
d_{22} \lambda_{1}^{k}+d_{23}\left(\begin{array}{c}
k \\
1
\end{array}\right) \lambda_{1}^{k-1}+\cdots+d_{2 n_{1}}\left(\begin{array}{c}
k \\
n_{1}-2
\end{array}\right) \lambda_{1}^{k-n_{1}+2} \\
\vdots \\
d_{n 2} \lambda_{1}^{k}+d_{n 3}\left(\begin{array}{c}
k \\
1
\end{array}\right) \lambda_{1}^{k-1}+\cdots+d_{n n_{1}}\left(\begin{array}{c}
k \\
n_{1}-2
\end{array}\right) \lambda_{1}^{k-n_{1}+2}
\end{array}\right], \ldots,\left(\begin{array}{c}
d_{1 n_{1}} \lambda_{1}^{k} \\
d_{2 n_{1}} \lambda_{1}^{k} \\
\vdots \\
d_{n n_{1}} \lambda_{1}^{k}
\end{array}\right]\right)
$$

and so on.

Thus, we have $A^{k}=\left[\begin{array}{ccc}\sum_{j=1}^{n_{1}} d_{1 j} n_{j 1} \lambda_{1}^{k}+\sum_{j=n_{1}+1}^{n_{2}} d_{1 j} n_{j 1} \lambda_{2}^{k}+\cdots & \cdots & \sum_{j=1}^{n_{1}} d_{1 j} n_{j n} \lambda_{1}^{k}+\sum_{j=n_{1}+1}^{n_{2}} d_{1 j} n_{j n} \lambda_{2}^{k}+\cdots \\ \vdots & \ddots & \vdots \\ \vdots & \\ \sum_{j=1}^{n_{1}} d_{n j} n_{j 1} \lambda_{1}^{k}+\sum_{j=n_{1}+1}^{n_{2}} d_{n j} n_{j 1} \lambda_{2}^{k}+\cdots & \cdots & \sum_{j=1}^{n_{1}} d_{n j} n_{j n} \lambda_{1}^{k}+\sum_{j=n_{1}+1}^{n_{2}} d_{n j} n_{j n} \lambda_{2}^{k}+\cdots\end{array}\right]$.

Let us assume that $\left|\lambda_{1}\right| \geq\left|\lambda_{2}\right| \geq \cdots \geq\left|\lambda_{r}\right|$. Then

$$
\lim _{k \rightarrow \infty} \frac{e^{T} A^{k+1} e}{e^{T} A^{k} e}=\lim _{k \rightarrow \infty} \frac{\sum_{i, j=1}^{n_{1}} d_{i j} n_{i j} \lambda_{1}^{k+1}+\sum_{i, j=n_{1}+1}^{n_{2}} d_{i j} n_{i j} \lambda_{2}^{k+1}+\cdots}{\sum_{i, j=1}^{n_{1}} d_{i j} n_{i j} \lambda_{1}^{k}+\sum_{i, j=n_{1}+1}^{n_{2}} d_{i j} n_{i j} \lambda_{2}^{k}+\cdots}
$$

Since $\lambda_{1}$ is the principal eigenvector of $A$ and $A y=\lambda_{1} y$, then $y=\lim _{m \rightarrow \infty} \frac{1}{m} \sum_{k=1}^{m} \frac{A^{k} e}{e^{T} A^{k} e}$ is the principal right eigenvector of $A$.

\section{An Example}

$$
A=\left(\begin{array}{cccc}
1 & 2 & 3 & 4 \\
1 / 2 & 1 & 5 & 6 \\
1 / 3 & 1 / 5 & 1 & 7 \\
1 / 4 & 1 / 6 & 1 / 7 & 1
\end{array}\right)
$$




$$
\begin{aligned}
& A^{2}=\left(\begin{array}{cccc}
1 & 4 & 9 & 16 \\
\frac{1}{4} & 1 & 25 & 36 \\
\frac{1}{9} & \frac{1}{25} & 1 & 7 \\
\frac{1}{16} & \frac{1}{36} & \frac{1}{49} & 1
\end{array}\right) \\
& A^{10}=\left(\begin{array}{cccc}
923637 . & 1.02324 \times 10^{6} & 2.52682 \times 10^{6} & 7.73336 \times 10^{6} \\
850356 . & 941992 . & 2.32613 \times 10^{6} & 7.11986 \times 10^{6} \\
369456 . & 409271 . & 1.01053 \times 10^{6} & 3.09298 \times 10^{6} \\
119539 . & 132434 . & 327011 & 1.00078 \times 10^{6}
\end{array}\right) \\
& A^{11}=\left(\begin{array}{cccc}
4.21087 \times 10^{6} & 4.66478 \times 10^{6} & 1.15187 \times 10^{7} & 3.52551 \times 10^{7} \\
3.87669 \times 10^{6} & 4.29457 \times 10^{6} & 1.06043 \times 10^{7} & 3.24561 \times 10^{7} \\
1.68418 \times 10^{6} & 1.86579 \times 10^{6} & 4.60711 \times 10^{6} & 1.41002 \times 10^{7} \\
544954 & 603711 & 1.49077 \times 10^{6} & 4.56261 \times 10^{6}
\end{array}\right) \\
& \frac{e^{T} A^{11} e}{e^{T} A^{10} e}=\frac{\frac{12727501858921621127}{93350880000}}{\frac{930627497489690773}{31116960000}}=\frac{12727501828921621127}{2791882492469072319}=4.558752692225857 \\
& \frac{e^{T} A^{21} e}{e^{T} A^{20} e}=\frac{\frac{16451807319718210925190569975581073}{31122809988480000000}}{\frac{7217595910655130158238943803833633}{6224561997696000000}}=\frac{32903614639436421850381139951162146}{7217595910655130158238943803833633}=4.55880532060014 \\
& 62245619976960000000
\end{aligned}
$$

Actual Eigenvalues of A

$$
\begin{aligned}
& \lambda_{1}=4.558805319078529 \\
& \lambda_{2}=-0.03996796194816203+1.583542975555991 a ̈ \\
& \lambda_{3}=-0.03996796194816203-1.583542975555991 a ̈ \\
& \lambda_{4}=-0.4788693951822055
\end{aligned}
$$

\section{References}

1. Hardy, G.H. Divergent Series, Oxford University Press, 1949.

2. Horn, R.A. and C.R. Johnson, Matrix Analysis, Cambridge University Press, 2012.

3. Saaty, T.L. and L.G. Vargas, Inconsistency and Rank Preservation, Journal of Mathematical Psychology, Vol. 28, No. 2, June 1984.

4. Saaty, T.L. The Analytic Hierarchy Process, McGraw Hill International, New York, 1980. Translated to Russian, Portuguese, and Chinese. Revised edition, paperback, RWS Publications, Pittsburgh, 1990, 1996.

5. Saaty, T.L. "New Light on the Theorem of Perron," Homenaje Al Professor Sixto Rios, Trabajos De Estadistica Y De Investigacion Operativa, Vol. 36, No. 3, 1985, pp. 253-257. 